Related content

\section{The connection between Dirac dynamic and parity symmetry}

To cite this article: C. H. Coronado Villalobos and R. J. Bueno Rogerio 2016 EPL 11660007

View the article online for updates and enhancements.
Non-standard Dirac adjoint spinor: The emergence of a new dual

R. J. Bueno Rogerio and C. H. Coronado Villalobos

A framework to a mass-dimension-one fermionic sigma mode

R. J. Bueno Rogerio, J. M. Hoff da Silva, S. H. Pereira et al.

Spin-half fermions with mass dimension one: theory, phenomenology, and darkmatter

D V Ahluwalia-Khalilova and D Grumiller

\section{Recent citations}

Type-4 spinors: transmuting from Elko to
$\frac{\text { single-helicity spinors }}{\text { C. H. Coronado Villalobos et al }}$
- The restricted Inomata-McKinley spinor-
$\frac{\text { plane, homotopic deformations and the }}{\text { Lounesto classification }}$
D. Beghetto et al
- Effective lagrangian for a mass dimension
one fermionic field in curved spacetime
R.J. Bueno Rogerio et al

R.J. Bueno Rogerio et al 


\title{
The connection between Dirac dynamic and parity symmetry
}

\author{
C. H. Coronado Villalobos and R. J. Bueno Rogerio
}

Departamento de Física e Química, Universidade Estadual Paulista - Guaratinguetá, SP, Brazil

received 22 November 2016; accepted in final form 19 January 2017

published online 8 February 2017

PACS 03.65.Pm - Relativistic wave equations

PACS $03.70 .+k$ - Theory of quantized fields

PACS 03.65.Fd - Algebraic methods

\begin{abstract}
Dirac spinors are important objects in the current literature, the algebraic structure presented in the text-books is a general method to write it, however, not unique. The purpose of the present work is to show an alternative approach to construct Dirac spinors, considering the interchange between the Lorentz representation space $(1 / 2,0)$ and $(0,1 / 2)$ made by the magic of Pauli matrices and not by parity, as was commonly thought. As is well known, the parity operator is related with the Dirac dynamics, as can be seen in SperançA L. D., Int. J. Mod. Phys. D, 2 (2014) 1444003. The major focus is to establish the relation between the Dirac dynamics with the parity operator, i.e., the reverse path shown in the paper by Sperança.

Copyright (c) EPLA, 2016
\end{abstract}

Introduction. - It is known that the full Lorentz group is composed by rotation generators, $\boldsymbol{J}$, boost generators, $\boldsymbol{K}$, and discrete symmetries: parity, $P$, and time reversion, or time reflection, $T$ [1]. The Dirac spinors are built within the full Lorentz group, keeping as the transformation law between the spinorial components, left-hand and right-hand components, the parity symmetry. In other words, this symmetry is exactly the link element between both parts of the representation space, i.e., it connects the subspaces $(j, 0)$ and $(0, j)$. In ref. [2] it was shown that parity operator, $P$, in the spin-( $1 / 2)$ representation, is given by

$$
P \psi(\boldsymbol{p})=m^{-1} \gamma_{\mu} p^{\mu} \psi(\boldsymbol{p})
$$

which allows us to derive the Dirac equation from the space-time symmetries alone.

Nevertheless, there are cases in which the representation spaces are not connected by discrete symmetries, like in the Elkos spinors case, proposed in ref. [3]. In this case the transformation law between the spinorial components is known as magic of Pauli matrices; this observation was first made by Ramond in [4], and it reads

$$
\Theta \sigma \Theta^{-1}=-\sigma^{*}
$$

where $\Theta$, in the spin-(1/2) representation, is given by

$$
\Theta=\left(\begin{array}{cc}
0 & -1 \\
1 & 0
\end{array}\right)
$$

The above operator is known as Wigner time reversal operator.

Following the Elko recipe, we present a set of single helicity spinors, which endows the magic of Pauli matrices as a crucial link between the representation space, for this case $(1 / 2,0)$ and $(0,1 / 2)$. The relevance of this work lies in the consequence of the fact that once imposed the Dirac dynamics for the spinors, it takes us directly to a relation between the phases and concomitantly the same relation is observed when it is imposed to the spinors being eigenspinors of the parity operator. On the other hand, if the spinors satisfy the Dirac dynamics, accordingly they are eigenspinors of the parity operator, and as was shown in [2], the inverse path is valid, too.

This paper is organized as follows: In the next section we define dual-helicity spinors as well single-helicity spinors and we present the right- and left-hand components and their respective helicity. In the third section we analyse the behaviour of the spinors under the action of the Dirac operator, via two distinct methods, and then the phases fixation to be coherent with the Dirac dynamics; finally we show that also it satisfies the Klein-Gordon equation. The fourth section is reserved to ascertain the action of parity symmetry over the spinors. We construct the dual spinor in the fifth section aiming to write the spin sums.

Proposing new spinors. - Motivated by the Elko theoretical discovery [5], we present here the construction of a spinor which inherits the same law of transformation 
between the spinorial components, or, in other words, the magic of the Pauli matrices. Following the same way of thought used in the Elko construction, we are able to obtain four spinors; two of them are dual-helicity objects and the remaining two are single-helicity objects. So, we start by showing the dual-helicity spinors. Basically these spinors essentially must be recognized as Elko. They are defined by

$$
\begin{aligned}
& \psi^{\{+,+\}}(\mathbf{0}) \equiv\left(\begin{array}{c}
\varsigma_{1} \Theta \phi_{L}^{*\{+\}}(\mathbf{0}) \\
\varsigma_{2} \Theta \phi_{R}^{*\{+\}}(\mathbf{0})
\end{array}\right), \\
& \psi^{\{-,-\}}(\mathbf{0}) \equiv\left(\begin{array}{c}
\varsigma_{1}^{\prime} \Theta \phi_{L}^{*\{-\}}(\mathbf{0}) \\
\varsigma_{2}^{\prime} \Theta \phi_{R}^{*\{-\}}(\mathbf{0})
\end{array}\right),
\end{aligned}
$$

where $\varsigma \in \mathbb{C}$ are arbitrary phases. For Elko spinors, the phases are fixed imposing $C \psi^{h}= \pm \psi^{h}[3]$. For the singlehelicity spinors, we have the following representation:

$$
\begin{aligned}
& \psi^{\{+,-\}}(\mathbf{0}) \equiv\left(\begin{array}{l}
\xi_{1} \Theta \phi_{L}^{*\{+\}}(\mathbf{0}) \\
\xi_{2} \Theta \phi_{R}^{*\{-\}}(\mathbf{0})
\end{array}\right), \\
& \psi^{\{-,+\}}(\mathbf{0}) \equiv\left(\begin{array}{l}
\xi_{1}^{\prime} \Theta \phi_{L}^{*\{-\}}(\mathbf{0}) \\
\xi_{2}^{\prime} \Theta \phi_{R}^{*\{+\}}(\mathbf{0})
\end{array}\right),
\end{aligned}
$$

the phase $\xi \in \mathbb{C}$ represents an arbitrary phase. Using the magic of the Pauli matrices allows to write, with no mention of discrete symmetries, a complete set of dualand single-helicity spinors. In the present work, our aim is to use this mathematical device to study the possibility to construct Dirac spinors, changing the usual link between the representation space $(1 / 2,0)$ and $(0,1 / 2)$, usually made via parity, but now made by relation (2). In this fashion, we look forward to understanding the physical content in this new set of spinors and to studying any consequence by the absent of discrete symmetry.

Choosing the components to be eigenstates of the helicity operator, $\vec{\sigma} \cdot \hat{p}$, one finds the left-hand spinorial componentes at the rest frame $[3,6]$,

$$
\begin{aligned}
& \phi_{L}^{\{+\}}(\mathbf{0})=\sqrt{m}\left(\begin{array}{c}
\cos (\theta / 2) e^{-i \phi / 2} \\
\sin (\theta / 2) e^{i \phi / 2}
\end{array}\right), \\
& \phi_{L}^{\{-\}}(\mathbf{0})=\sqrt{m}\left(\begin{array}{c}
-\sin (\theta / 2) e^{-i \phi / 2} \\
\cos (\theta / 2) e^{i \phi / 2}
\end{array}\right),
\end{aligned}
$$

and the right-hand components as well

$$
\begin{aligned}
& \phi_{R}^{\{+\}}(\mathbf{0})=\sqrt{m}\left(\begin{array}{c}
-i \sin (\theta / 2) e^{-i \phi / 2} \\
i \cos (\theta / 2) e^{i \phi / 2},
\end{array}\right), \\
& \phi_{R}^{\{-\}}(\mathbf{0})=\sqrt{m}\left(\begin{array}{c}
-i \cos (\theta / 2) e^{-i \phi / 2} \\
-i \sin (\theta / 2) e^{i \phi / 2}
\end{array}\right) .
\end{aligned}
$$

The mass term is chosen such that in the massless limit the rest spinors in the representation space $(1 / 2,0)$ and $(0,1 / 2)$ identically vanish, so that there can be no massless particle at rest [7]. The interaction amplitudes must have the factor $m^{j}$, where $j$ is the spin of the particle, in order for the $m \rightarrow 0$ limit be consistent [8].

Regarding the helicity, we have to described the action of the operator $\vec{\sigma} \cdot \hat{p}$ at the spinorial components. Thus, we can define the following relations:

$$
\begin{aligned}
& \vec{\sigma} \cdot \hat{p} \Theta \phi_{L}^{*\{ \pm\}}(\mathbf{0})=\mp \Theta \phi_{L}^{*\{ \pm\}}(\mathbf{0}), \\
& \vec{\sigma} \cdot \hat{p} \Theta \phi_{R}^{*\{ \pm\}}(\mathbf{0})= \pm \Theta \phi_{R}^{*\{ \pm\}}(\mathbf{0}) .
\end{aligned}
$$

Such relationships are of great importance and will be used for the scope of this work. Once we had defined above the action of the operator $\vec{\sigma} \cdot \hat{p}$ on the rest frame spinors, one is able to write the spinors in a boosted frame with an arbritary momentum. So, the boosted spinors are given by the action of the boost matrix, as we can see below

$$
\psi^{\{h,-h\}}(\boldsymbol{p})=\left(\begin{array}{cc}
\kappa^{(1 / 2,0)} & 0 \\
0 & \kappa^{(0,1 / 2)}
\end{array}\right) \psi^{\{h,-h\}}(\mathbf{0})
$$

where the boost matrix components $\kappa^{(1 / 2,0)}$ and $\kappa^{(0,1 / 2)}$ are the right-hand and left-hand boost operators, respectively. They are defined by $[3,9]$

$$
\begin{aligned}
\kappa^{(1 / 2,0)} & =\sqrt{\frac{E+m}{2 m}}\left(\mathbb{I}+\frac{\vec{\sigma} \cdot \vec{p}}{E+m}\right), \\
\kappa^{(0,1 / 2)} & =\sqrt{\frac{E+m}{2 m}}\left(\mathbb{I}-\frac{\vec{\sigma} \cdot \vec{p}}{E+m}\right) .
\end{aligned}
$$

In this way, the boosted-frame dual-helicity spinors are given by

$$
\begin{aligned}
& \psi^{\{+,+\}}(\boldsymbol{p})=\sqrt{\frac{E+m}{2 m}}\left(1-\frac{p}{E+m}\right) \psi^{\{+,+\}}(\mathbf{0}), \\
& \psi^{\{-,-\}}(\boldsymbol{p})=\sqrt{\frac{E+m}{2 m}}\left(1+\frac{p}{E+m}\right) \psi^{\{-,-\}}(\mathbf{0}),
\end{aligned}
$$

and for the single-helicity case we have

$$
\psi^{\{h\}}(\boldsymbol{p})=\sqrt{\frac{E+m}{2 m}}\left(\begin{array}{cc}
\mathbb{I}+p \frac{\vec{\sigma} \cdot \hat{p}}{E+m} & 0 \\
0 & \mathbb{I}-p \frac{\vec{\sigma} \cdot \hat{p}}{E+m}
\end{array}\right) \psi^{\{h\}}(\mathbf{0}),
$$

where $h$ stands for $\{ \pm, \mp\}$ helicities. So, in this manner, it is possible to define all the possible spinors in an arbitrary momentum inertial frame. The Dirac equation can be derived by simply appealing to the properties of the parity operator $P$. So we define such operator to be

$$
P \psi(\boldsymbol{p})=\eta \psi(-\boldsymbol{p}),
$$

where $\eta$ is an off-diagonal matrix

$$
\eta=\left(\begin{array}{ll}
0 & \mathbb{I} \\
\mathbb{I} & 0
\end{array}\right)
$$


Then, using (19) and (20), we easily obtain

$$
P=m^{-1} \gamma_{\mu} p^{\mu}
$$

The above operator has eigenvalues $\pm 1[2]$. Indeed, the focus of this paper is to find spinors governed by the Dirac dynamic and then verify its behaviour under the parity operator, showing the inverse path and the correspondence between the mentioned method and the method analysed by Sperança in ref. [2].

Field dynamics. - In this section, our focus is to verify the dynamic associated to the proposed spinors. The set of spinors belongs to the Lorentz proper orthochronous group, formed only by rotation and boost generators; for this reason the focus is now to evaluate any emergent consequences, if any, carried by the mentioned fact, in the dynamical interpretation. For this task we start analysing whether this set of spinors satisfies the Dirac equation and how the phases must be related and fixed. Finally, we shall evaluate if it satisfies the Klein-Gordon equation, as a consistent check.

In order to verify if all the spinors satisfy the Dirac equation, we employ here two distinct methods. We start by using the same mathematical construction that was made in [10] and lastly the second method is the action of the Dirac operator, $\gamma_{\mu} p^{\mu}$, upon the spinors.

Here we start using the same formalism used in [10], where the Dirac operator is built starting from a rest frame spinor and then acting with the boost operator, from (14) it is known that

$$
\begin{aligned}
& \psi^{\{+,-\}}(\boldsymbol{p})=\sqrt{\frac{E+m}{2 m}} \\
& \times\left(\begin{array}{cc}
\mathbb{I}+p \frac{\vec{\sigma} \cdot \hat{p}}{E+m} & 0 \\
0 & \mathbb{I}-p \frac{\vec{\sigma} \cdot \hat{p}}{E+m}
\end{array}\right) \psi^{\{+,-\}}(\mathbf{0}) .
\end{aligned}
$$

For didactic purposes, as an example, we use $\psi^{\{+,-\}}(\boldsymbol{p})$ (see footnote ${ }^{1}$ )

$\psi^{\{+,-\}}(\boldsymbol{p})=\sqrt{\frac{E+m}{2 m}}\left(\begin{array}{l}\left(\mathbb{I}+p \frac{\vec{\sigma} \cdot \hat{p}}{E+m}\right) \xi_{1} \Theta \phi_{L}^{*\{+\}}(\mathbf{0}) \\ \left(\mathbb{I}-p \frac{\vec{\sigma} \cdot \hat{p}}{E+m}\right) \xi_{2} \Theta \phi_{R}^{*\{-\}}(\mathbf{0})\end{array}\right)$,

where we can easily identify the right-hand component as

$$
\phi_{R}^{\{+\}}(\boldsymbol{p})=\frac{E+m+\vec{\sigma} \cdot \vec{p}}{\sqrt{2 m(E+m)}} \xi_{1} \Theta \phi_{L}^{*\{+\}}(\mathbf{0}),
$$

with the left-hand component

$$
\phi_{L}^{\{-\}}(\boldsymbol{p})=\frac{E+m-\vec{\sigma} \cdot \vec{p}}{\sqrt{2 m(E+m)}} \xi_{2} \Theta \phi_{R}^{*\{-\}}(\mathbf{0}) .
$$

${ }^{1}$ In the present approach we use only $\psi^{\{+,-\}}(\boldsymbol{p})$, to simplify the calculations. The algorithm is identical to the other spinors.
As the next step, we present some useful relations

$$
\begin{aligned}
& \Theta \phi_{L}^{*\{+\}}(\mathbf{0})=-i \Theta \phi_{R}^{*\{-\}}(\mathbf{0}), \\
& \Theta \phi_{R}^{*\{-\}}(\mathbf{0})=i \Theta \phi_{L}^{*\{+\}}(\mathbf{0}) .
\end{aligned}
$$

Inserting the last two relations above in eqs. (25) and (26), it is easy to find

$$
\phi_{R}^{\{+\}}(\boldsymbol{p})=-i \xi_{1} \xi_{2}^{*}\left[\frac{E+\vec{\sigma} \cdot \vec{p}}{m}\right] \phi_{L}^{\{-\}}(\boldsymbol{p}),
$$

and

$$
\phi_{L}^{\{-\}}(\boldsymbol{p})=i \xi_{1}^{*} \xi_{2}\left[\frac{E-\vec{\sigma} \cdot \vec{p}}{m}\right] \phi_{R}^{\{+\}}(\boldsymbol{p}) .
$$

The formalism presented in these calculations shows that eqs. (29) and (30) allow to write them in a simple matricial form,

$$
\left(\begin{array}{cc}
m & -i \xi_{1} \xi_{2}^{*}\left(p_{0}+\vec{\sigma} \cdot \vec{p}\right) \\
i \xi_{1}^{*} \xi_{2}\left(p_{0}-\vec{\sigma} \cdot \vec{p}\right) & -m
\end{array}\right)\left(\begin{array}{c}
\phi_{R}^{\{+\}}(\boldsymbol{p}) \\
\phi_{L}^{\{-\}}(\boldsymbol{p})
\end{array}\right)=0
$$

In order for the last equation to be regarded as a Dirac equation, we must fix the phases as

$$
\xi_{1}=i \xi_{2}
$$

With this condition, eq. (31) becomes

$$
\left(\begin{array}{cc}
-m & \left(p_{0}+\vec{\sigma} \cdot \vec{p}\right) \\
\left(p_{0}-\vec{\sigma} \cdot \vec{p}\right) & -m
\end{array}\right)\left(\begin{array}{c}
\phi_{R}^{\{+\}}(\boldsymbol{p}) \\
\phi_{L}^{\{-\}}(\boldsymbol{p})
\end{array}\right)=0,
$$

making it possible to write (33) as

$$
\left(\gamma_{\mu} p^{\mu}-m \mathbb{I}\right) \psi^{\{+,-\}}(\boldsymbol{p})=0 .
$$

Repeating the same procedure for $\psi^{\{-,+\}}(\boldsymbol{p})$, we conclude

$$
\xi_{1}^{\prime}=-i \xi_{2}^{\prime}
$$

in this manner

$$
\left(\gamma_{\mu} p^{\mu}-m \mathbb{I}\right) \psi^{\{-,+\}}(\boldsymbol{p})=0 .
$$

The last equation provides to the reader that all $\psi(\boldsymbol{p})$ satisfy the Dirac equation, as far as eq. (35) holds. Applying once again the same procedure in eq. (36), it becomes easy to verify

$$
\left(\square+m^{2}\right) \psi^{\{ \pm, \mp\}}(\boldsymbol{p})=0,
$$

which reproduces the Klein-Gordon equation annihilating all $\psi^{h}(\boldsymbol{p})$.

As said before, the second method consists in analysing spinors under the action of the $\gamma_{\mu} p^{\mu}$ operator, taking care of the conditions that govern the phases terms. In this 
way, we operate with $\gamma_{\mu} p^{\mu}$ upon the spinors in (6), thus

$$
\begin{aligned}
& \gamma_{\mu} p^{\mu} \psi^{\{+,-\}}(\boldsymbol{p})= \\
& {\left[E \gamma_{0}+\left(\begin{array}{cc}
0 & p \vec{\sigma} \cdot \hat{p} \\
-p \vec{\sigma} \cdot \hat{p} & 0
\end{array}\right)\right] \psi^{\{+,-\}}(\boldsymbol{p}) .}
\end{aligned}
$$

Using eqs. (12) and (23), one is able to write the spinor in a boosted frame with an arbitrary momentum as $^{2}$

$$
\psi^{\{+,-\}}(\boldsymbol{p})=\left(\begin{array}{l}
\xi_{1} \Lambda_{-}\left(p^{\mu}\right) \Theta \phi_{L}^{*\{+\}}(\mathbf{0}) \\
\xi_{2} \Lambda_{+}\left(p^{\mu}\right) \Theta \phi_{R}^{*\{-\}}(\mathbf{0})
\end{array}\right) .
$$

Now, operating with $\gamma_{\mu} p^{\mu}$ over (39) we have

$$
\gamma_{\mu} p^{\mu} \psi^{\{+,-\}}(\boldsymbol{p})=\left(\begin{array}{c}
(E-p) \xi_{2} \Lambda_{+}\left(p^{\mu}\right) \Theta \phi_{R}^{*\{-\}}(\mathbf{0}) \\
(E+p) \xi_{1} \Lambda_{-}\left(p^{\mu}\right) \Theta \phi_{L}^{*\{+\}}(\mathbf{0})
\end{array}\right)
$$

and we can see that $\psi^{\{+,-\}}(\boldsymbol{p})$ is not an eigenspinor of $\gamma_{\mu} p^{\mu}$. It is easy to see that the matricial components of eq. (40) is shifted when compared to (39). To continue with the calculations, we use the following trick, actually, we are using the relations between $\Theta$ and the spinorial leftand right-hand components, given in eqs. (27) and (28).

Taking that into account and using the last two relations above, one is able to write

$$
\gamma_{\mu} p^{\mu} \psi^{\{+,-\}}(\boldsymbol{p})=i m\left(\begin{array}{c}
\xi_{2} \Lambda_{-}\left(p^{\mu}\right) \Theta \phi_{L}^{*\{+\}}(\mathbf{0}) \\
-\xi_{1} \Lambda_{+}\left(p^{\mu}\right) \Theta \phi_{R}^{*\{-\}}(\mathbf{0}),
\end{array}\right) .
$$

Firstly, for $\psi^{\{+,-\}}(\boldsymbol{p})$, eq. (41) only represents the Dirac equation, if the following conditions are imposed over the phases:

$$
\xi_{1}=+i \xi_{2} \quad \text { and } \quad \xi_{2}=-i \xi_{1},
$$

and for $\psi^{\{-,+\}}(\boldsymbol{p})$, the relation must be set as

$$
\xi_{1}^{\prime}=-i \xi_{2}^{\prime} \quad \text { and } \quad \xi_{2}^{\prime}=+i \xi_{1}^{\prime}
$$

which is in agreement with first method, thus, showing the consistency between both methods. As was expected, the spinors must to satisfy the Klein-Gordon equation, then we easily conclude

$$
\left(\square+m^{2}\right) \psi^{\{+,-\}}(\boldsymbol{p})=0 .
$$

Obviously the last equation is satisfied by the other remaining spinors. Thereby, we have shown that both methods work and are consistent.

Defining a complete set of spinors via parity operator. - In order to build a complete set for the $\psi$ spinors, we introduce here the action of the parity operator, as in the Dirac case. In the $(1 / 2,0) \oplus(0,1 / 2)$ representation space, the parity operator reads

$$
P=e^{i \Phi} \gamma_{0} \mathcal{R},
$$

\footnotetext{
${ }^{2}$ In order to summarize the notation we define the boosts operators so that it reads $\Lambda_{ \pm}\left(p^{\mu}\right) \equiv \sqrt{\frac{E+m}{2}}\left(1 \pm p \frac{\sigma \cdot \hat{p}}{E+m}\right)$.
}

where the $\mathcal{R}$ acts as

$$
\mathcal{R} \equiv\{\theta \rightarrow \pi-\theta, \phi \rightarrow \phi+\pi, p \rightarrow p\},
$$

and we consider an arbitraty phase $e^{i \Phi}$, which will be fixed if necessary. Thus, we act with the parity operator over $\psi^{\{-,+\}}(\boldsymbol{p})$,

$$
P \psi^{\{-,+\}}(\boldsymbol{p})=e^{i \Phi} \gamma_{0} \mathcal{R}\left(\begin{array}{l}
\xi_{1}^{\prime} \Lambda_{+}\left(p^{\mu}\right) \Theta \phi_{L}^{*\{-\}}(\mathbf{0}) \\
\xi_{2}^{\prime} \Lambda_{-}\left(p^{\mu}\right) \Theta \phi_{R}^{*\{+\}}(\mathbf{0})
\end{array}\right) .
$$

Analysing the action of $\mathcal{R}$ over the spinors components, we have

$$
\begin{aligned}
& \mathcal{R} \phi_{L}^{*\{-\}}(\boldsymbol{p})=\phi_{L}^{*\{-\}}(\boldsymbol{p}), \\
& \mathcal{R} \phi_{R}^{*\{+\}}(\boldsymbol{p})=\phi_{R}^{*\{+\}}(\boldsymbol{p}) .
\end{aligned}
$$

Finally, eq. (47) can be expresed as

$$
P \psi^{\{-,+\}}(\boldsymbol{p})=e^{i \Phi}\left(\begin{array}{c}
-i \xi_{2}^{\prime} \Lambda_{+}\left(p^{\mu}\right) \Theta \phi_{L}^{*\{-\}}(\mathbf{0}) \\
i \xi_{1}^{\prime} \Lambda_{-}\left(p^{\mu}\right) \Theta \phi_{R}^{*\{+\}}(\mathbf{0})
\end{array}\right) .
$$

In order that the spinor in eq. (50) becomes an eigenspinor of the parity operator, we impose

$$
\xi_{2}^{\prime}=i \xi_{1}^{\prime} e^{i \Phi}
$$

and for the other case, $\psi^{\{+,-\}}(\boldsymbol{p})$, reads

$$
\xi_{2}=-i \xi_{1} e^{i \Phi},
$$

fixing $e^{i \Phi}=+1$ we have positive eigenvalues, so it is easy then to conclude $P \psi_{+}^{\{\lambda\}}=+\psi_{+}^{\{\lambda\}}$. Now, imposing $\psi$ to have negative eigenvalues, we must fix $e^{i \Phi}=-1$, in other words, $P \psi_{-}^{\{\lambda\}}=-\psi_{-}^{\{\lambda\}}$, then, for the phases of $\psi^{\{+,-\}}(\boldsymbol{p})$ we have the condition ${ }^{3}$

$$
\xi_{4}=i \xi_{3},
$$

and for the other case, $\psi^{\{-,+\}}(\boldsymbol{p})$, it reads

$$
\xi_{4}^{\prime}=-i \xi_{3}^{\prime}
$$

So, we are able to write a complete set of single-helicity spinors:

$$
\begin{aligned}
& \psi_{+}^{\{+,-\}}(\boldsymbol{p})=\left(\begin{array}{c}
\xi_{1} \Lambda_{-}\left(p^{\mu}\right) \Theta \phi_{L}^{\{+\} *}(\mathbf{0}) \\
-i \xi_{1} \Lambda_{+}\left(p^{\mu}\right) \Theta \phi_{R}^{\{-\} *}(\mathbf{0})
\end{array}\right), \\
& \psi_{+}^{\{-,+\}}(\boldsymbol{p})=\left(\begin{array}{c}
\xi_{1}^{\prime} \Lambda_{+}\left(p^{\mu}\right) \Theta \phi_{L}^{\{-\} *}(\mathbf{0}) \\
i \xi_{1}^{\prime} \Lambda_{-}\left(p^{\mu}\right) \Theta \phi_{R}^{\{+\} *}(\mathbf{0})
\end{array}\right),
\end{aligned}
$$

both spinors above with positive eigenvalue, and analogously

$$
\begin{aligned}
& \psi_{-}^{\{+,-\}}(\boldsymbol{p})=\left(\begin{array}{c}
\xi_{3} \Lambda_{-}\left(p^{\mu}\right) \Theta \phi_{L}^{\{+\} *}(\mathbf{0}) \\
i \xi_{3} \Lambda_{+}\left(p^{\mu}\right) \Theta \phi_{R}^{\{-\} *}(\mathbf{0})
\end{array}\right), \\
& \psi_{-}^{\{-,+\}}(\boldsymbol{p})=\left(\begin{array}{c}
\xi_{3}^{\prime} \Lambda_{+}\left(p^{\mu}\right) \Theta \phi_{L}^{\{-\} *}(\mathbf{0}) \\
-i \xi_{3}^{\prime} \Lambda_{-}\left(p^{\mu}\right) \Theta \phi_{R}^{\{+\} *}(\mathbf{0})
\end{array}\right),
\end{aligned}
$$

${ }^{3}$ To avoid confusion and to elucidate that the phases are different from those in the previous analysis, we consider $\xi_{1} \rightarrow \xi_{3}$ and $\xi_{2} \rightarrow$ $\xi_{4}$, the same being still valid for the primed phases. 
with negative eigenvalue. In this context we defined a complete set of eigenspinors of the parity operator, the last two remaining spinors $\psi_{-}^{h}(\boldsymbol{p})$, given in eqs. (57) and (58), both satisfying the dynamics presented in the previous section. It is our duty to point out that spinors in eqs. (55)(58) have a non-unitary transformation, in this sense, they form a basis playing the role of expansion coefficients of a quantum field.

Dual construction and spin sums. - In the second section, we have constructed all the possible spinors using the components given in eqs. (8) and (10), the dualhelicity spinors and the single-helicity spinors. Now, in this present section, our next task is to use the defined single-helicity spinors in eq. (6) to find the corresponding dual (eventually check if it is the same dual as for the Dirac case as shown in [10]). For this task we shall apply the same mathematical prescription, a mathematically rigorous recipe, as adopted recently for the Elko dual construction at $[11,12]$, which can be followed to construct or define any dual. The quantity $\widetilde{\psi}^{h}(\boldsymbol{p}) \psi^{h}(\boldsymbol{p})$ must yield an invariant and real definite norm, in addition it must secure a positive definite norm for two $\psi^{h}$, and negative-definite norm for the other remaining two. Any other choice becomes unjustifiable and forbids a physical and relevant interpretation. After some calculations, we easily conclude

$$
\begin{aligned}
& \widetilde{\psi}_{ \pm}^{\{+,-\}}(\boldsymbol{p})=\left[\psi_{ \pm}^{\{+,-\}}(\boldsymbol{p})\right]^{\dagger} \gamma_{0}, \\
& \widetilde{\psi}_{ \pm}^{\{-,+\}}(\boldsymbol{p})=\left[\psi_{ \pm}^{\{-,+\}}(\boldsymbol{p})\right]^{\dagger} \gamma_{0} .
\end{aligned}
$$

The dual spinor obtained is the same as for the Dirac case. With the dual thus defined, we have now, by construction, the orthonormality relations given by

$$
\begin{aligned}
& \widetilde{\psi}_{+}^{h}(\boldsymbol{p}) \psi_{+}^{h^{\prime}}(\boldsymbol{p})=+2 m \delta^{h h^{\prime}}, \\
& \widetilde{\psi}_{-}^{h}(\boldsymbol{p}) \psi_{-}^{h^{\prime}}(\boldsymbol{p})=-2 m \delta^{h h^{\prime}}, \\
& \widetilde{\psi}_{ \pm}^{h}(\boldsymbol{p}) \psi_{\mp}^{h^{\prime}}(\boldsymbol{p})=0 .
\end{aligned}
$$

Thus, we have defined the dual structure that provides a complete set of orthonormality relations ensuring a possible quantization.

The spin sums play a very important role in the quantum field, they give information about the locality structure, fermionic statistics and information about the Feynman-Dyson propagator structure, since this quantity appears on the core of the quantum field operator. A particular aspect about the spin sums is that in some cases they are proportional to the wave operator, as happens in the Dirac case $[3,10]$. So, here we will write the spin sums using the dual structure calculated, in order to obtain relevant information about the field locality structure. Now, we have a particular case, where the spinor dual structure matches Dirac's dual. Therefore, is important to evaluate the spin sums, and then verify if this amount has the same structure as it has for Dirac's case. So, we have

$$
\sum_{h=\{ \pm, \mp\}} \psi_{+}^{h}(\boldsymbol{p}) \widetilde{\psi}_{+}^{h}(\boldsymbol{p})=\left(\gamma_{\mu} p^{\mu}+m \mathbb{I}\right),
$$

and

$$
\sum_{h=\{ \pm, \mp\}} \psi_{-}^{h}(\boldsymbol{p}) \widetilde{\psi}_{-}^{h}(\boldsymbol{p})=\left(\gamma_{\mu} p^{\mu}-m \mathbb{I}\right) .
$$

Analogously to the Dirac spinors in [10], $\psi_{+}^{h}(\boldsymbol{p})$ can be interpreted as the bi-spinors $u(\boldsymbol{p})$ wave function and following the same reasoning for $\psi_{-}^{h}(\boldsymbol{p})$, which can be interpreted as $v(\boldsymbol{p})$ wave functions.

In the standard model of particle physics, all the regarded spinors are of Dirac, Weyl or Majorana type. Such spinors obey a first-order derivative field equation. This characteristic implies a quantum propagator that, for large momentum, is proportional to $p^{-1}$. This asymptotic behaviour of the associated propagator results, among other things, in the fact that mass dimension must be $3 / 2$. Now, the unique kinematic operator that is satisfied by Elko is the Klein-Gordon equation, a second-order derivative field equation. For this case, for a large momentum, the quantum propagator is proportional to $1 / p^{2}$, contradicting the previous case. The Klein-Gordon operator is the proper kinetic operator for Elko fields. We are, therefore, led to conclude that the mass dimension of the Elko field is 1 , rather than $3 / 2$, as would be usually expected for a fermionic field [13].

Lounesto's spinors classification and generalizations. - Consider the Minkowski spacetime $\left(M, \eta_{\mu \nu}\right)$ and its tangent bundle $T M$. Denoting sections of the exterior bundle by $\sec \Lambda(T M)$, given a $k$-vector $a \in \sec \Lambda^{k}(T M)$, the reversion is defined by $\tilde{a}=(-1)^{|k / 2|} a$, whilst the grade involution reads $\hat{a}=(-1)^{k} a$, where $|k|$ stands for the integral part of $k$. By extending the Minkowski metric from $\sec \Lambda^{1}(T M)=\sec T^{*} M$ to $\sec \Lambda(T M)$, and considering $a_{1}, a_{2} \in \sec \Lambda(V)$, the left contraction is given by $\left.g(a\lrcorner a_{1}, a_{2}\right)=g\left(a_{1}, \tilde{a} \wedge a_{2}\right)$. Classical spinors are objects of the space that carry the usual $\tau=(1 / 2,0) \oplus(0,1 / 2)$ representation of the Lorentz group, that can be thought of as being sections of the vector bundle $\mathbf{P}_{\operatorname{Spin}_{1,3}^{e}}(M) \times_{\tau} \mathbb{C}^{4}$.

Given a spinor field $\psi \in \sec \mathbf{P}_{\operatorname{Spin}_{1,3}^{e}}(M) \times_{\tau} \mathbb{C}^{4}$, the bilinear covariants are sections of the bundle $\Lambda(T M)[14,15]$. Indeed, the well-known Lounesto's spinors classification is based upon bilinear covariants and the underlying multivector structure. The physical nature of the classification focuses on the bilinear covariants, that are physical observables, characterizing the types of fermionic particles. The observable quantities are given by the following multivectors:

$$
\begin{array}{rlrl}
\sigma & =\psi^{\dagger} \gamma_{0} \psi, & \omega & =-\psi^{\dagger} \gamma_{0} \gamma_{0123} \psi, \\
J_{\mu} & =\psi^{\dagger} \gamma_{0} \gamma_{\mu} \psi, & K_{\mu}=\psi^{\dagger} \gamma_{0} i \gamma_{0123} \gamma_{\mu} \psi, \\
S_{\mu \nu} & =\frac{1}{2} \psi^{\dagger} \gamma_{0} i \gamma_{\mu \nu} \psi, & &
\end{array}
$$

where $\gamma_{5}=i \gamma_{0} \gamma_{1} \gamma_{2} \gamma_{3}$. The set $\left\{\mathbf{1}, \gamma_{I}\right\}$ (where $I \in\{\mu, \mu \nu, \mu \nu \rho, 5\}$ is a composed index) is a basis for $\mathcal{M}(4, \mathbb{C})$ satisfying $\gamma_{\mu} \gamma_{\nu}+\gamma_{\nu} \gamma_{\mu}=2 \eta_{\mu \nu} \mathbf{1}$. 
The above bilinear covariants in the Dirac theory are interpreted, respectively, as the mass of the particle $(\sigma)$, the pseudo-scalar $(\omega)$ relevant for parity-coupling, the current of probability $(\mathbf{J})$, the direction of the electron spin $(\mathbf{K})$, and the probability density of the intrinsic electromagnetic moment $(\mathbf{S})$ associated to the electron. The most important bilinear covariant for our goal here is $\mathbf{J}$, although with a different meaning. A prominent requirement for Lounesto's spinors classification is that the bilinear covariants satisfy quadratic algebraic relations, namely, the so-called Fierz-Pauli-Kofink (FPK) identities, which read

$$
\begin{aligned}
J_{\mu} J^{\mu} & =\sigma^{2}+\omega^{2}, & & J_{\mu} J^{\mu}=-K_{\mu} K^{\mu}, \\
J_{\mu} K^{\mu} & =0, & & \mathbf{J} \wedge \mathbf{K}=-\left(\omega+\sigma \gamma_{0123}\right) \mathbf{S} .
\end{aligned}
$$

It is worth remarking that the above identities are fundamental, not merely for the aims regarding the classification, but also for asserting the inversion theorem. Within the Lounesto classification scheme, a nonvanishing $\mathbf{J}$ is crucial, since it enables to define the socalled boomerang [14] which has an ample geometrical meaning to assert that there are precisely six different classes of spinors. This is a prominent consequence of the definition of a boomerang [14]. As far as the boomerang is concerned, it is not possible to exhibit more than six types of spinors, according to the bilinear covariants. Indeed, Lounesto's spinor classification splits regular and singular spinors. The regular spinors are those which have at least one of the bilinear covariants $\sigma$ and $\omega$ non-null. On the other hand, singular spinors present $\sigma=0=\omega$, and in this case the Fierz identities are in general replaced by the more general conditions [15]

$$
\begin{aligned}
Z^{2} & =4 \sigma Z, \quad Z \gamma_{\mu} Z=4 J_{\mu} Z, \quad Z \gamma_{0123} Z=-4 \omega Z, \\
Z i \gamma_{\mu \nu} Z & =4 S_{\mu \nu} Z, \quad Z i \gamma_{0123} \gamma_{\mu} Z=4 K_{\mu} Z .
\end{aligned}
$$

When an arbitrary spinor $\eta$ satisfies $\widetilde{\eta^{*}} \psi \neq 0$ and belongs to $\mathbb{C} \otimes \mathcal{C} \ell_{1,3}$-or equivalently when $\eta^{\dagger} \gamma_{0} \psi \neq 0 \in$ $\mathcal{M}(4, \mathbb{C})$ - it is possible to recover the original spinor $\psi$ from its aggregate $\mathbf{Z}$ given by

$$
\mathbf{Z}=\sigma+\mathbf{J}+i \mathbf{S}+i \mathbf{K} \gamma_{0123}+\omega \gamma_{0123}
$$

and the spinor $\eta$ by the so-called Takahashi algorithm [16] likewise. In fact, the spinor $\psi$ and the multivector $\mathbf{Z} \eta$ differ solely by a multiplicative constant, and can be thus written as

$$
\psi=\frac{1}{2 \sqrt{\eta^{\dagger} \gamma_{0} \mathbf{Z} \eta}} e^{-i \theta} \mathbf{Z} \eta,
$$

where $e^{-i \theta}=2\left(\eta^{\dagger} \gamma_{0} \mathbf{Z} \eta\right)^{-1 / 2} \eta^{\dagger} \gamma_{0} \psi \in U(1)$. The Takahashi algorithm reveals the importance of the aggregate. From the geometric point of view, the following relations between the bilinear covariants must be fulfilled in order to ensure that the aggregate be a boomerang: J must be parallel to $\mathbf{K}$ and both are in the plane formed by the bivector S. Hence, using eq. (67) and taking into account that we are dealing with singular spinors, it is straightforward to see that the aggregate can be recast as

$$
\mathbf{Z}=\mathbf{J}\left(1+i \mathbf{s}+i h \gamma_{0123}\right),
$$

where $\mathbf{s}$ is a space-like vector orthogonal to $\mathbf{J}$, and $h$ is a real number. The multivector as expressed in eq. (69) is a boomerang [17]. By inspecting the condition $Z^{2}=4 \sigma Z$ we see that for singular spinors $\mathbf{Z}^{2}=0$. However, in order for the FPK identities to hold it is also necessary that both conditions ${ }^{4} \mathbf{J}^{2}=0$ and $\left(\mathbf{s}+h \gamma_{0123}\right)^{2}=-1$ must be satisfied. These considerations are important in order to constrain the possible spinor classes.

Now, let us explicit that from (68) one can see that different bilinear covariants combinations may lead to different spinors, taking into account the constraints coming from the FPK identities. Altogether, the algebraic constraints reduce the possibilities to six different spinor classes, namely:

1) $\sigma \neq 0, \quad \omega \neq 0$;

2) $\sigma \neq 0, \quad \omega=0$;

3) $\sigma=0, \quad \omega \neq 0$;

4) $\sigma=0=\omega, \quad \mathbf{K} \neq 0, \quad \mathbf{S} \neq 0$

5) $\sigma=0=\omega, \quad \mathbf{K}=0, \quad \mathbf{S} \neq 0$;

6) $\sigma=0=\omega, \quad \mathbf{K} \neq 0, \quad \mathbf{S}=0$.

The spinors types (1), (2) and (3), are called Dirac spinor fields (regular spinors). The spinor field (4) is called flagdipole, while the spinor field (5) is named flag-pole [18]. Majorana [19] and Elko [3,17] spinors are elements of the flag-pole class. Finally, the type-(6) dipole spinors are examplified by Weyl spinors. Note that there are only six different spinor fields. To see that, notice that for the regular case, since $\mathbf{J} \neq 0$, it follows that $\mathbf{S} \neq 0$ and $\mathbf{K} \neq 0$ as impositions from the identities (65). On the other hand, for the singular case, the geometry asserts that $\mathbf{J}\left(\mathbf{s}+h \gamma_{0123}\right)=\mathbf{S}+\mathbf{K} \gamma_{0213}$. Aiming to classify the proposed spinors within the Lounesto classification, to illustrate this general case, we use the spinor $\psi^{\{+,-\}}(\boldsymbol{p})$ with an arbitrary phase, so in this way one has the 16 bilinear forms given by

$$
\begin{aligned}
\sigma & =i m\left(\xi_{1}^{*} \xi_{2}-\xi_{1} \xi_{2}^{*}\right), \\
\omega & =-m\left(\xi_{1}^{*} \xi_{2}+\xi_{1} \xi_{2}^{*}\right), \\
J_{0} & =2 E, \\
J_{1} & =-2 p \sin (\theta) \cos (\phi), \\
J_{2} & =-2 p \sin (\theta) \sin (\phi), \\
J_{3} & =2 p \cos (\theta), \\
K_{0} & =-2 p, \\
K_{1} & =2 E \sin (\theta) \cos (\phi),
\end{aligned}
$$

${ }^{4}$ We remark that $\mathbf{J}$ must be different from zero in the Lounesto classification. 


$$
\begin{aligned}
K_{2} & =2 E \sin (\theta) \sin (\phi), \\
K_{3} & =-2 E \cos (\theta), \\
S_{01} & =i m / 2\left(\xi_{1}^{*} \xi_{2}+\xi_{1} \xi_{2}^{*}\right) \sin (\theta) \cos (\phi), \\
S_{02} & =m / 2\left(\xi_{1}^{*} \xi_{2}+\xi_{1} \xi_{2}^{*}\right) \sin (\theta) \sin (\phi), \\
S_{03} & =m / 2\left(\xi_{1}^{*} \xi_{2}+\xi_{1} \xi_{2}^{*}\right) \cos (\theta), \\
S_{12} & =i m / 2\left(\xi_{1} \xi_{2}^{*}-\xi_{1}^{*} \xi_{2}\right) \cos (\theta), \\
S_{13} & =i m / 2\left(\xi_{1} \xi_{2}^{*}-\xi_{1}^{*} \xi_{2}\right) \sin (\theta) \sin (\phi), \\
S_{23} & =i m / 2\left(\xi_{1} \xi_{2}^{*}-\xi_{1}^{*} \xi_{2}\right) \sin (\theta) \cos (\phi) .
\end{aligned}
$$

Therefore, without phase fixation this spinor is classified as type (1) which matches the Dirac spinor. Now, for the case where we impose that spinors have the same dynamic as the Dirac spinors, i.e., $\xi_{1}=i \xi_{2}$, we easily find the following set of real bilinear forms which satisfies the Fierz identities, given in (65), With these considerations, we conclude that the proposed spinor with phases fixed, belongs to type (2) within the Lounesto classification. The last result shows the possibility to construct Dirac spinors without reference to the parity operator.

Final remarks. - The purpose of this work was to show the possibility to construct Dirac spinors, analogously as was made by Elko, however, in abstaining parity symmetry. It should be mentioned, that in this context, we do not consider parity symmetry as a crucial link between the Lorentz representation spaces $(1 / 2,0)$ and $(0,1 / 2)$, although this symmetry arises from the requirement to construct a complete set of spinors, such as $u(\boldsymbol{p})$ and $v(\boldsymbol{p})$, for Dirac spinors. It is important to comment about this feature, since we constructed the reverse path contrasting with the work [2]. Basically, we claim that independently of choosing $P$ symmetry or the dynamics given $\gamma_{\mu} p^{\mu}$, choosing one another comes naturally, and vice versa. Both are always correlated independently of the choice.

Guided by the classification for the spinors developed by Lounesto in [14], the spinors proposed here are classified as Dirac type (2), when the phases are given by relations (42) and (43), as was expected. On the other hand, if the phases are fixed as $\xi_{1}= \pm i$ and $\xi_{2}=\mp i$, we obtain spinors that do not satisfy the Dirac dynamic but belong to type (3) within the Lounesto classification.

We believe that parity symmetry is not a unique link between representation spaces, we have shown here that the absence of such link does not affect the field dynamic, it remains the same as for the Dirac case. In this way, to conclude this discussion, we have shown that if and only if the spinor satisfies the Dirac dynamic it is an eigenspinor of the parity operator, being a counterpart for what was made in [2].

Finally, it is important to emphasize that the only similarity between the Elko and the Dirac spinors constructed here is given by the magic of Pauli matrices as a link for the representation spaces, whilst the crucial differences are single helicity, mass dimension 3/2, Lorentz-invariant spin sums and Lounesto classification, contrasting with the Elko dual-helicity feature, mass dimension 1, noninvariant spin sums and the Elko spinors do not belong to the Lounesto classification, as can be seen in detail in [20].

The authors express their gratitude to Prof. Julio MARny Hoff DA Silva for the privilege of his revision, comments and appreciation of this work. CHCV acknowledges PEC-PG for the financial support. RJBR acknowledges CAPES for the financial support.

\section{REFERENCES}

[1] WeinBerg S., The Quantum Theory of Fields, first edition, Vol. 1, (Cambridge University Press, New York) 1995.

[2] SperançA L. D., Int. J. Mod. Phys. D, 2 (2014) 1444003.

[3] Ahluwalia D. V. and Grumiller D., JCAP, 07 (2005) 012.

[4] Ramond P., Field Theory: A Modern Primer, second edition (Addison Wesley-Publishing, Cal. USA) 1989.

[5] Ahluwalia D. V. and Grumiller D., Phys. Rev. D, 72 (2005) 067701.

[6] Bhmer C. G. and Corpe L., J. Phys. A, 45 (2012) 205206.

[7] Ahluwalia D. V., Johnson M. B. and Goldman T., Mod. Phys. Lett. A, 9 (1994) 439.

[8] Marinov M. S., Ann. Phys., 49 (1968) 357.

[9] Ahluwalia D. V., Lee Cheng-Yang and Schritt D., Phys. Lett. B, 687 (2010) 248.

[10] Ryder L. H., Quantum Field Theory, second edition (Cambridge University Press, New York) 1996.

[11] Ahluwalia D. V., On a local mass dimension one Fermi field of spin one-half and the theoretical crevice that allows it, arXiv:1305.7509 [hep-th] (2013).

[12] Ahluwalia D. V., Lee Cheng-Yang and Schritt D., Phys. Rev. D, 83 (2011) 065017.

[13] Bueno Rogerio R. J., Hoff da Silva J. M., Pereira S. H. and DA Rocha R., EPL, 113 (2016) 60001.

[14] Lounesto P., Clifford Algebras and Spinors, second edition (Cambridge University Press, Cambridge) 2002.

[15] Crawford J. P., J. Math. Phys., 26 (1985) 1439.

[16] Takahashi Y., Phys. Rev. D, 26 (1982) 2169.

[17] da Rocha R. and Hoff da Silva J. M., Adv. Appl. Clifford Algebras, 20 (2010) 847.

[18] BenN I. M. and TuCKer R. W., An Introduction to Spinors and Geometry with Applications in Physics (Adam Hilger, Bristol) 1987.

[19] Majorana E., Nuovo Cimento, 9 (1932) 43.

[20] Hoff da Silva J. M., Coronado Villalobos C. H., Bueno Rogerio R. J. and Scatena E., Eur. Phys. J. $C, \mathbf{7 6}$ (2016) 563. 